\title{
De Tristes Tropiques a Paroles d'un Chaman Yanomani, la collection Terre Humaine de Jean Malaurie Chez Plon : une grande collection de sciences sociales a l'ecoute du Monde
}

\author{
Jean-Yves Mollier \\ Université de Versailles Saint-Quentin-en-Yvelines \\ Centre d'histoire culturelle des sociétés contemporaines
}

La collection Terre Humaine dirigée par Jean Malaurie fêtera en 2014 ses soixante années d'existence sous la direction du même homme qui, en 1955, donnait à lire Tristes Tropiques, le reportage ethnographique de Claude Lévi-Strauss paru dans cette bibliothèque. Lui-même venait d'y publier, quelques mois plus tôt Les Derniers Rois de Thulé, ouvrage dans lequel il alertait le monde sur les dangers qui menaçaient les peuples Inuit du nord du Groenland. La traduction des Carnets indiens de Darcy Ribeiro, en 2002, fut un événement, de même que l'avait été la réédition, en 1998, du livre publié quarante ans auparavant par Roger Bastide, Le candomblé de Bahia. En 2010, la sortie d'un volume intitulé La chute du ciel. Paroles d'un chaman Yanomani de Davi Kopenawa, le leader des Indiens du Brésil, confirme l'intérêt demeuré intact de l'editor de la collection la plus prestigieuse des sciences humaines françaises pour un pays dont son ami Claude Lévi-Strauss lui avait précocement montré l'étonnante richesse et révélé la diversité culturelle. Si l'on ajoute que cette remarquable bibliothèque a aussi inscrit Les veines ouvertes de l'Amérique latine d'Eduardo Galeano à son catalogue en 1981, qu'elle a fait connaitre les mœurs des Urubu anthropophages du Nord du Brésil, avec Aimables Sauvages, en 1960, et celles des populations de l'Amazonie en publiant, en 1968, Yanoama, le récit d'Helena Valero qui vécut vingt-deux ans dans cette région, sans compter la Chronique des Indiens Guayaki du Paraguay rapportée par Pierre Clastres en 1972, on comprend que l'on est en présence d'une collection tout à fait exceptionnelle qui mérite qu'on en retrace la genèse et l'inscription dans le paysage scientifique européen ${ }^{1}$. 
Les bibliothèques, entendues au sens de dépôts de manuscrits puis de livres, ont une longue histoire, que symbolisent la disparition puis la reconstruction récente de celle d'Alexandrie en Egypte. La publication des Règles du bibliothécaire par les bénédictins de Saint-Maur, la grande abbaye parisienne, en 1663, marque une date et annonce une autre approche de la répartition des fonds documentaires, plus proche de nos préoccupations. Toutefois il faut parvenir à la toute fin du XVIIe siècle pour voir naitre d'authentiques collections de livres portant le nom de 'bibliothèques', ce qui ajoute au sens initial celui qui va s'imposer pour ce que l'on peut appeler les 'bibliothèques sans toit ou sans murs'. Roger Chartier en a recensé trente-et-une pour la période qui s'étend de 1686 à $1789^{2}$, date qui marquera une rupture puisque le grand siècle de la collection sera le XIXe ${ }^{3}$, avant que l'apparition du livre de poche, au suivant, n'en démultiplie les occasions ${ }^{4}$. Parmi les plus célèbres, on citera la Bibliothèque Charpentier ${ }^{5}$ apparue en 1838 et la Bibliothèque des chemins de fer de Louis Hachette ${ }^{6}$, née elle en 1853, parce que l'une et l'autre correspondent à une massification du lectorat et à la mise à la portée du plus grand nombre de livres à bas prix, $3 \mathrm{~F} 50$ ou 15 euros dans le premier cas et $1 \mathrm{~F}$ ou 5 euros dans le second. Très vite, à côté des collections de romans qui sont monnaie courante dans les années 1830-1870, d'autres séries voient le jour, telle la Bibliothèque des merveilles d'Edouard Charton ${ }^{7}$ en 1864 qui unit la féérie à la vulgarisation scientifique.

Dans le domaine des sciences humaines, il faudra attendre l'après-Seconde Guerre mondiale pour voir surgir de véritables collections ordonnées selon des principes cohérents car, chez Albert Savine ou Pierre-Victor Stock, au tournant des XIXe et XXe siècle, une Bibliothèque sociologique réunit encore tous ceux qui entendent contester ou réorganiser la société, Bakounine, Kropotkine, Louise $\mathrm{Michel}^{8}$, ce qui ne peut manquer de surprendre le lecteur du XXIe siècle, habitué à d'autres classements de la pensée, pour ne pas dire à d'autres représentations mentales. Tandis qu'à Paris les Presses Universitaires de France, nées en 1921 et restructurées en 1939 après leur absorption de trois fonds d'édition qui ont gonflé leur catalogue, lancent en 1941 la collection encyclopédique Que sais-je ?", les éditeurs de littérature générale commencent, au début des années 1950, à se ranger en ordre de bataille pour affronter l'univers mouvementé des sciences sociales. L'essor du marxisme, celui du structuralisme, la notoriété de Jean-Paul Sartre et de l'existentialisme, les 
progrès fulgurants de la sociologie ainsi que de l'anthropologie nourrissent la curiosité du public cultivé qui n'entend pas demeurer à l'écart des débats publics. De grandes revues, Esprit, Les Temps modernes, Critique, La Pensée, relaient les discussions et les controverses qui ont lieu dans le milieu académique $^{10}$, ce qui explique pourquoi, dans les années 1970, Roland Barthes, Michel Foucault, Louis Althusser, Jacques Derrida et quelques autres grands intellectuels pourront apparaître aux yeux de l'étranger comme les pères de la French Theorie ${ }^{11}$. Les Brésiliens n'avaient pas attendu l'après-guerre pour se familiariser avec les travaux de la jeune école de sciences sociales et ils seront parmi les premiers à traduire les volumes parus dans la collection Terre Humaine dont il convient maintenant d'examiner la spécificité.

\section{Terre Humaine chez Plon, un phénomène typiquement français}

On s'attendrait en effet à voir cette bibliothèque spécialisée apparaitre au catalogue d'un éditeur situé près de la Sorbonne, de la faculté de médecine ou des jeunes instituts de recherche qui se développent après guerre, la VIe section de l'Ecole Pratique des Hautes Etudes, la matrice de l'Ecole des Hautes Etudes en Sciences Sociales, par exemple, mais il n'en est rien et c'est au contraire chez 'une vieille dame endormie', l'austère et très maurrassienne maison Plon, l'éditeur des Mémoires de Churchill, de Guderian, du général De Gaulle et du maréchal Joukov que Terre Humaine verra le jour fin $1954^{12}$. Jean Malaurie en a plusieurs fois conté le miraculeux et incongru avènement chez un éditeur que rien ne destinait à cette intrusion dans le domaine de l'anthropologie mais qui avait bien des raisons de faire oublier ses complaisances envers le régime de Vichy, quitte, pour cela, à faire appel à des jeunes trublions, du moment qu'ils ne flirtaient pas trop ostensiblement avec le marxisme. A la Libération en effet, le champ éditorial français avait subi de graves perturbations. Les nouveaux venus dans ce milieu, telles les Editions de Minuit de Vercors, celles du Seuil, d'Emmanuel Mounier, ou les maisons fondées par Edmond Charlot, le premier éditeur d'Albert Camus, et quelques autres émanaient de la Résistance et ces nouveaux venus étaient bien décidés à purger la profession des brebis galeuses qui avaient accepté trop facilement la loi allemande et le nazisme. Bernard Grasset allait y laisser son entreprise, Robert Denoël sa vie, et Gaston Gallimard craignit un moment de payer 
les conséquences de l'entrée de Drieu La Rochelle à la direction de la $\mathrm{NrF}$. Quant à la société Plon, Nourrit et Cie, très compromise avec Vichy, elle se rallia tardivement aux nouvelles autorités et elle boira le calice jusqu'à la lie quand elle éditera les Mémoires de guerre du général de Gaulle, elle qui avait publié le maréchal Pétain et ne jurait que par la défense des valeurs incarnées par la Révolution nationale que le premier avait combattue ${ }^{13}$.

Heureusement pour ses dirigeants, l'homme politique qui avait incarné la France Libre en 1940 allait rapidement quitter le pouvoir après avoir refusé, début 1946, de continuer à diriger un gouvernement dans lequel le Parti Communiste était tout puissant et il allait apparaitre aux yeux de la droite de son pays comme le rempart contre la révolution, ce qui ne pouvait que rassurer et même séduire Maurice Bourdel, le PDG de la société. Pour faire taire ses détracteurs et amadouer les juges qui devaient examiner l'attitude de sa firme pendant la guerre, il avait embauché fin 1944 Charles Orengo, un résistant de la dernière heure qui, lui aussi, voulait occulter le fait qu'il avait été rémunéré comme censeur par le régime de Vichy et qui y était parvenu en s'attribuant un faux brevet de résistant emprisonné pour ses idées ${ }^{14}$. Intelligent, à l'affût de la nouveauté, grand lecteur et véritable éditeur au sens de dénicheur de talents non encore éclos, c'est lui qui accueillit en 1954 un jeune explorateur de retour d'expédition dans le grand Nord, Jean Malaurie, qui lui fit un récit très excitant de sa rencontre avec les Inuit. L'idée de lui confier une collection de livres qui aurait un caractère scientifique incontestable et une qualité d'écriture qui l'apparenterait à la fiction avait germé dans son cerveau en l'écoutant. Puisque la course au loin attirait les lecteurs depuis le milieu du XIXe siècle et que de nombreux magazines, du genre Le Tour $d u$ monde et le Journal des voyages avaient préparé le terrain de multiples séries de romans dont Les voyages extraordinaires de Jules Verne, chez Hetzel, étaient l'exemple type, rien ne s'opposait à la recréation d'un espace éditorial pour ce type de littérature ${ }^{15}$. Dans un après-guerre où la conquête spatiale n'était pas encore à l'ordre du jour et où la guerre froide et l'affrontement des deux grands faisaient craindre le pire, offrir une tribune à des aventuriers dotés d'un bagage scientifique suffisant pouvait être une excellente initiative.

La rencontre entre Jean Malaurie et les vieillards affublés de sonotones qui composaient, selon sa malicieuse reconstitution, le conseil d'administration des éditions Plon, déboucha sur un contrat limité aux quelques livres qui 
trouvèrent grâce à leurs yeux, Les Derniers Rois de Thulé et Tristes Tropiques en 1955, Les Immémoriaux de Segalen en 1956, Afrique ambiguë de Georges Balandier en 1957 et Soleil hopi de Don C. Talayesva en 1959. Avec cinq volumes portés au catalogue en cinq ans, le résultat était maigre et il convient donc d'éviter de proposer une réécriture hagiographique d'une collection qui, manifestement, n'enthousiasmait pas les propriétaires ni le gérant de la maison Plon mais qui conservait la confiance du directeur, soucieux de dépoussiérer la firme et de faire venir à elle des auteurs qui en renouvelleraient le fonds ${ }^{16}$. Dans la mesure où les deux premiers titres avaient été bien accueillis par la critique et où des traductions étaient en chantier, la maison d'édition ne pouvait que se féliciter du flair de Charles Orengo. Pour la sortie de Tristes Tropiques, elle avait même vu le jury du Prix Goncourt se fendre d'un communiqué pour expliquer qu'il regrettait que le règlement de leur académie impose de récompenser un roman car, s'ils avaient été libres de leur vote, les jurés auraient couronné le livre de Claude Lévi-Strauss. Rappeler les circonstances exactes de la publication de ce classique de l'ethnologie, c'est souligner la parenté générique de cette étude avec le reportage et même la fiction. Jean Malaurie avait insisté auprès de son collègue pour qu'il lui donne autre chose qu'une thèse ou qu'une brochure destinée aux milieux savants. Lui-même s'était volontiers plié à cette règle lorsqu'il avait raconté son expérience et sa vie parmi les Inuit. En proposant un récit capable de tenir le lecteur en haleine, il savait qu'il prenait le risque de déplaire aux mandarins qui dirigeaient la Sorbonne mais il était conscient du fait qu'un succès public et une presse élogieuse ne manqueraient pas d'influer sur le jugement de leurs pairs.

On pénètre par ce biais dans la fabrique éditoriale de la collection «Terre Humaine » et l'on voit que même si elle se situe bien dans le domaine des sciences sociales, elle s'en distingue par certaines caractéristiques qui l'apparentent au roman et expliquent pourquoi c'est une maison d'édition dite de littérature générale qui l'abrita et non une entreprise spécialisée située au pôle non commercial du champ. Jean Malaurie voulait comme Pierre Nora en histoire ou Michel Foucault en psychologie, sa discipline universitaire initiale, briser les codes en vigueur et pénétrer avec fracas dans la citadelle académique et ils y parvinrent à la faveur des circonstances, la France de la Quatrième République se prêtant à ces mutations du champ intellectuel. La massification de 
l'enseignement supérieur, la montée des classes moyennes, la croissance économique, tous ces facteurs contribuaient au changement et comme il existait des précédents célèbres, la Vie de Jésus de Renan ou Le Peuple de Michelet, tous deux professeurs au Collège de France mais également auteurs de livres qui s'étaient très bien vendus et avaient servi leur notoriété, les éditeurs et les milieux savants ne pouvaient s'indigner de voir ce mélange des genres ou cette confusion des univers perturber leurs habitudes. Au début du XXe siècle, on avait même vu un médecin sans véritables diplômes, Gustave Le Bon, diriger chez Flammarion la Bibliothèque de philosophie scientifique et attirer chez cet éditeur très grand public tout ce que l'Université comptait comme célébrités académiques. Loin de s'apparenter aux collections publiées par les presses universitaires de Cambridge, d'Oxford ou de Columbia, de Harvard, de Princeton ou de Yale, Terre Humaine se situait ainsi d'emblée dans une tradition française à mi-chemin entre le public savant et le public cultivé, ce qui ne pouvait que contribuer à sa médiatisation.

Un catalogue représentatif des curiosités de son directeur

Quand on feuillette le catalogue de la collection Terre Humaine ou qu'on regarde l'étude qu'en a tirée, pour la période 1955-2000, Pierre Aurégan, et qu'il a intitulée Des récits et des hommes. Terre Humaine : un autre regard sur les sciences de l'homme, on vérifie le caractère éclectique d'une bibliothèque sans frontières autres que celles que lui fixe l'humeur vagabonde de son directeur. Un comptage rapide des titres qui composent la série montre aussi que, contrairement à ce qu'il a pu écrire ou dire, Jean Malaurie n'a pas eu les coudées aussi franches qu'il l'aurait souhaité. Il a d'ailleurs souvent reconnu que Maurice Bourdel ne voulait pas de Victor Segalen chez lui parce qu'il le jugeait invenda$b^{18}{ }^{18}$. Avec cinq titres publiés les cinq premières années puis dix volumes les dix suivantes, Terre Humaine ne comptait que quinze titres en 1969 et vingt-cinq en 1975, ce qui la condamnait presque immanquablement à la disparition. Aucune autre collection en effet, qu'elle relève des sciences exactes ou des sciences sociales, de la fiction ou d'un autre genre, ne peut justifier son existence quand elle ne parvient pas à dépasser un titre par an en moyenne. C'est le succès prodigieux du Cheval d'orgueil, le récit d'un professeur breton qui faisait revivre, avec un vrai talent de conteur, le passé de sa région, ses traditions, ses croyances 
et son patrimoine menacés par la modernité, qui devait stabiliser définitivement la présence de Jean Malaurie chez Plon et même lui assurer désormais le statut envié de directeur de collection rapportant de l'argent à son éditeur. L'époque se prêtait à la narration et, chez Fayard, Les Nouvelles Etudes historiques venaient d'être relancées au moment où elles allaient disparaître, grâce à la popularité d'une biographie, celle de Louis XI, que Paul Murray Kendall avait opportunément sorti de l'oubli. En utilisant les ressorts de Walter Scott et en donnant de l'épaisseur à ses personnages, il prouvait à ceux qui en doutaient que l'histoire pouvait encore faire rêver et que le champ scientifique n'était pas un domaine réservé aux spécialistes ${ }^{19}$.

La liste des vingt-cinq premiers ouvrages inscrits au catalogue de Terre Humaine laisse rêveur si on replace leur présence dans une maison d'édition particulièrement marquée politiquement et toujours nostalgique du régime de Vichy. La lecture d'Afrique ambiguë de Georges Balandier ne pouvait que heurter les conceptions de dirigeants patronaux pour qui la colonisation avait écrit une des pages les plus glorieuses de l'histoire de France et qui n'éprouvaient que mépris envers les leaders indigènes qui aspiraient à prendre le pouvoir et à se substituer à l'homme blanc. Certes l'auteur n'était pas connu comme un agitateur ni un ethnologue particulièrement engagé mais son livre ne laissait aucun doute sur ses préférences et ses certitudes quant à l'avenir du continent noir. De même Terres vivantes de René Dumont, publié en 1961, s'il n'est pas encore un hymne à l'écologie, décrit avec sympathie les paysans du tiers monde que l'agronome a croisés pendant ses voyages à travers la planète. Deux ans plus tard, Jean Malaurie lançait un pavé dans la mare en offrant, en traduction, quatre études de l'anthropologue Margaret Mead, qui, bien avant les travaux de Judith Butler, risquaient d'introduire le trouble dans le genre puisque son livre, Mours et sexualité en Océanie, soulignait la relativité des rôles masculins et féminins dans certaines populations. Certes l'auteur était devenue la référence par excellence en matière d'anthropologie mais si des lecteurs commençaient à imaginer que ce qui était admis dans les sociétés océaniennes pourrait l'être un jour en Occident, la maison Plon passerait pour une officine dangereuse, ce qui faisait heureusement sourire ceux qui lisaient les déclarations de ses dirigeants, toujours soucieux de respecter la morale et de ne publier que des auteurs comme François Mauriac, Paul Claudel ou Gabriel Marcel ${ }^{20}$. 
Comme devait le dire publiquement Maurice Bourdel à la journaliste qui l'interrogeait pour Les Nouvelles littéraires en 1952 : "Nous avons toujours en vue une sorte de rassemblement des écrivains de talent ayant un idéal commun de défense de la civilisation occidentale" et, pour être sûr d'être bien compris, il ajoutait : "En fait, la ligne de la maison est très simple : encourager et faire connaître les écrivains de qualité, soucieux de défendre les couleurs éternelles de la civilisation humaniste et chrétienne, au sens le plus élevé et le plus large du terme" ${ }^{21}$. De façon un peu étonnante, il en concluait que cela prédisposait sa maison d'édition à recruter les jeunes auteurs et les originaux et il citait en vrac Marguerite Yourcenar, pour Les Mémoires d'Hadrien, François Mauriac et Henri Troyat ainsi que Mouloud Mammeri, mais ce dernier était solidement encadré par le médecin eugéniste Alexis Carrel dont Plon avait vendu par centaines de milliers d'exemplaires L'Homme, cet inconnu, Mazo de La Roche pour la série des Jalna et le Roumain anticommuniste Virgil Gheorghiu dont La Vingt-cinquième heure avait eu un énorme retentissement en 1949. Sorte de pendant aux révélations d'Arthur Koestler qui, dans Le Zéro et l'Infini, paru chez Calmann-Lévy, avait dénoncé les perversités du système soviétique, le récit de Gheorgiu illustrait la volonté des éditions Plon, Nourrit et Cie de demeurer sur des positions idéologiques que la plupart des volumes retenus pas Jean Malaurie contestaient. La réaction épidermique de Maurice Bourdel face au roman de Victor Segalen, Les Immémoriaux, traduit bien son refus d'admettre que le christianisme et la colonisation aient pu, même dans une région aussi reculée que la Polynésie, s'acharner à détruire une civilisation aussi belle que celle des Maoris.

Un village anatolien de Mahmout Akal ou Un substitut de campagne en Egypte de Tewfik El Hakim devaient poser moins de problèmes parce que, dans ces deux enquêtes, l'Islam était décrit sans complaisance mais la maison Plon se reconnaissait mal dans la plupart des titres qui, année après année, renforçaient le catalogue de "Terre Humaine " et en faisaient véritablement une collection à part dans le domaine des sciences humaines. L'attirance pour les sociétés rurales ne déplaisait pas particulièrement à une firme tournée vers le passé mais Soleil hopi, de Don C. Talayaesva, Ishi, de Theodora Kroeber, Piegan de Richard Lancaster,et Louons maintenant les grands hommes de James Agee pour le texte et Walker Evans pour les photographies étaient à cent lieues des préoccupations de dirigeants patronaux qui siégeaient depuis des années 
dans les conseils d'administration de leur syndicat, qui avaient appartenu au Comité d'organisation du Livre mis en place par les services du maréchal Pétain et qui ne s'étaient réconciliés avec la démocratie qu'en voyant le général de Gaulle, après 1958, attaquer durement la gauche et le Parti communiste avec qui il avait frayé en 1944-1945. L'entrée de Jacques Soustelle, en 1967, pour Les Quatre Soleils, était plus rassurante à leurs yeux car l'ethnologue qui avait été résistant était passé du gaullisme militant à l'Organisation Armée secrète, après 1961, et avait dû s'exiler pour échapper à la prison ${ }^{22}$. Du coup, c'est le camp progressiste qui comprit mal pourquoi Jean Malaurie accueillait dans sa collection un homme qui s'était totalement éloigné de ses études mexicaines et qui semblait profiter de l'occasion pour réhabiliter, sans le dire, sa conception figée de l'évolution des sociétés humaines.

Deux autres livres avaient certainement choqué chez Plon : Des affaires de famille de Francis A.J. Ianni, un reportage sociologique sur la mafia aux Etats-Unis dans lequel l'auteur s'interdisait tout jugement de valeur, et Leurs Prisons de Bruce Jackson, ouvrage préfacé par Michel Foucault et consacré à l'univers carcéral américain. Plus que le sujet qui pouvait être accepté, c'est la parole donnée aux 'tapettes, lopes et macs' dans la troisième partie du volume qui ne passait pas. Jean Malaurie avait loisir d'invoquer la tradition du roman français, Balzac qui, dans Splendeurs et misères des courtisanes, avait mis en scène la pègre et les galériens, ou Jean Genet et son Journal d'un voleur. Toutefois ce dernier était précisément un auteur Gallimard accusé de favoriser les homosexuels, brutalement dénoncés comme des 'pédérastes', et jamais Plon n’aurait songé à le faire entrer dans la maison que le général de Gaulle avait préférée à Gallimard et à Robert Laffont, en 1958, quand il s'était agi de publier le premier volume de ses Mémoires de guerre. Gardienne d'une tradition et, en ce sens, plus soucieuse que d'autres, de maintenir une cohérence idéologique dans son catalogue, la maison Plon, Nourrit et Cie ne ressemblait pas à ses jeunes concurrentes qui ne cherchaient qu'à faire parler d'elles, comme la maison Julliard qui lancera Françoise Sagan, ou les Presses de la Cité qui lorgnaient sur l'Amérique pour préparer des collections tape-à-l'œil susceptibles de plaire aux lecteurs transformés en simples consommateurs. Indépendante et familiale, riche et sans souci matériel avant 1960, la firme implantée à deux pas de l'église et du séminaire Saint-Sulpice avait même investi des fonds dans le démarrage des 'Editions' de La Table Ronde, une entreprise 
située très à droite de l'échiquier politique ${ }^{23}$, ce qui explique qu'à la veille de la publication du Cheval d'orgueil, en 1975, la situation de Jean Malaurie ait empiré au sein d'une société pour qui Terre Humaine faisait figure d'hapax ou d'ovni, c'est-à-dire de collection totalement à part qui n'avait d'autre justification que le désir des dirigeants de conserver un pied dans un secteur où elles ne pouvaient rivaliser ni avec les PUF, ni avec le Seuil ou Gallimard.

Toutefois, Maurice Bourdel s'était retiré sans héritier mâle et il avait cédé sa société à une banque qui la revendit au PDG des Presses de la Cité, Sven Nielsen, un Danois qui avait réalisé de juteux profits avec la collection Le Fleuve noir et qui, profitant des circonstances, était en train de se tailler un empire dans le petit monde de l'édition française du milieu des années 1960. Ayant racheté Plon, Perrin, Julliard, 10-18 et bien d'autres maisons d'édition, il laissait les hommes qu'il avait recrutés pour le seconder diriger ses sociétés et Jean Malaurie bénéficia d'un répit qui lui permit de continuer à publier les auteurs qu'il aimait ${ }^{24}$. Charles Orengo avait été brutalement débarqué et celui qui le remplaça, Marcel Jullian, était un romancier doublé d'un scénariste de télévision qui, quoique royaliste, ne laissait pas ses sentiments dicter ses décisions. Poussé par le financier propriétaire à rentabiliser la nouvelle entité juridique, Plon-Perrin, il aurait sans doute été amené à suspendre Terre Humaine et à laisser la collection mourir de sa belle mort, si le succès, on l'a dit, du 'Cheval d'orgueil', ne l'avait convaincu que l'inclassable et original Jean Malaurie pouvait encore se montrer capable de faire parler de lui et de la maison d'édition qui l'hébergeait depuis vingt ans. En ce sens, les circonstances pesèrent d'un grand poids en 1975 pour pérenniser une bibliothèque scientifique absolument hors normes qui, comme ses homologues des sciences humaines, était menacée par le succès des collections de poche et, notamment, par «10-18», une série dont le nom évoque les petites dimensions (dix centimètres sur dix-huit) et, partant, le bas prix. Plébiscitée par les étudiants qui, après 1968, achètent systématiquement ses volumes, ceux des Essais chez Gallimard, Point Histoire au Seuil ou leurs équivalents, cette collection que dirige alors Christian Bourgois appartient aussi à Sven Nielsen et elle aurait pu si on regarde de près son catalogue introduire la plupart des titres de Terre Humaine dans son fonds si les 300000 exemplaires du livre de Pierre-Jakez Hélias, Le cheval d'orgueil, ne l'avaient momentanément sauvée ${ }^{25}$. 


\section{Terre humaine de 1975 à l'aube du IIIe millénaire}

Le catalogue comportait vingt-cinq titres en 1975 et soixante-et-un en 1995, ce qui signifie que l'augmentation n’a pas été très importante puisque la moyenne annuelle n'atteint qu'à peine deux volumes et il faut donner raison à Jean-Claude Dubost qui, lors du colloque du cinquantenaire, en 2005, rendait à la fois hommage à la perspicacité de Charles Orengo et à celle de Bernard de Fallois, le nouveau PDG qui, en 1982, décida de doubler Terre Humaine par la collection Terre Humaine Poche qui lui donna une assurance qu'elle n'avait jamais possédée antérieurement ${ }^{26}$. Entre-temps, L'Eté grec de Jacques Lacarrière, Le Grand Métier - sur la pêche à la morue - Toinou d'Antoine Sylvère - l'histoire d'un métayer auvergnat - et Gaston Lucas, serrurier d'Adélaïde Blasquez avaient ajouté aux chroniques de la planète et aux carnets d'enquête des ethnologues le récit de la vie des simples, un pêcheur, un berger ou un serrurier rejoignant le paysan dans la peinture de la grandeur du quotidien. Incontestablement, ces choix constituent bien l'horizon personnel du directeur et confèrent à sa collection une unité que nul ne songerait à lui contester. Refusant le culte du progrès, la conquête spatiale et tous les hymnes entonnés par d'autres aux constructeurs de barrages ou aux ingénieurs qui révolutionnent alors les habitudes et les modes de vie des hommes, Jean Malaurie se situe, diront certains, à l'avant-garde de ce qui fera la chair du mouvement écologiste dans les années 1980, ou à l'arrière-garde diront d'autres en observant que ses héros sont essentiellement des ruraux, des marginaux ou des exclus de la civilisation. Sensibilisé très tôt à la disparition programmée des peuples les plus faibles, Jean Malaurie a conféré à ses livres cette coloration qui en fait, non des ouvrages militants, encore moins de propagande, mais des témoignages, accessibles au plus grand nombre, et des invitations à méditer l'évolution de nos sociétés ${ }^{27}$. Ni devant ni derrière mais décalé, l'anthropologue ami des Inuit donne la parole en ces années 1970-1980 à ceux qui lui paraissent capables de dire des vérités dérangeantes et de décrire avec humilité leur vie et celle de ceux qui les entourent.

Ouvrant de plus en plus le compas, le directeur de la collection Terre Humaine donne à lire des récits de vie rurale concernant la Mésopotamie avec Les Arabes des marais de Wilfred Thesiger, la paysannerie hongroise la plus pauvre et la plus éloignée de la culture écrite, avec Le vinaigre et le fiel 
de Marget Gari, mais il revient à ses thèmes favoris en redonnant la parole à Bruce Jackson, avec Le Quartier de la mort, à René Dumont, avec Pour l'Afrique, j'accuse dont le titre explique peut-être pourquoi le volume suivant sera les Carnets d'enquête d'Emile Zola qui se vendra au-delà des espérances de son préfacier, Henri Mitterand. Le Horsain, itinéraire d'un prêtre étranger au pays de Caux où il est affecté, inaugure une écoute des problèmes qui traversent l'Eglise catholique et qui débouchera sur un grand livre, Quand Rome condamne, de François Leprieur, un ancien prêtre ouvrier sommé, comme ses frères, de choisir entre demeurer dans l'Eglise et renoncer à son apostolat à l'usine ou conserver son nouveau métier et s'interdire l'accès aux sacrements. Mineur de fond d'Augustin Viseux, sorte de Germinal du XXe siècle mais écrit par un porion, donc un agent de maîtrise et non un ouvrier, rencontrera lui aussi les faveurs du public. D’autres volumes, consacrés au Japon - La Maison Yamazaki de Laurence Caillet - au Sud tunisien, Chebika de Jean Duvignaud - ou Un village russe témoignent de la variété des paysages qui entrent les uns après les autres dans une série qui semble se donner pour tâche d'enfermer la diversité du monde physique et de capter le regard de toutes les populations et de tous les groupes humains menacés de disparition plus ou moins proche ${ }^{28}$.

Très présent depuis le deuxième volume, Tristes Tropiques, le Brésil est sans aucun doute le pays le mieux représenté dans cette collection puisqu'après Aimables Sauvages de Francis Huxley, en 1960, Yanoama d'Ettore Biocca en 1968, Chronique des Indiens guayaki de Pierre Clastres en 1972, Les veines ouvertes de l'Amérique latine d'Eduardo Galeano en 1981, ces deux derniers titres débordant le cadre du seul Brésil, Les lances du crépuscule de Philippe Descola en 1994, Le Candomblé de Bahia en 1999 forment un ensemble important de sept livres consacrés aux Indiens de l'Amazonie ou aux populations qui habitent à l'intérieur des frontières du Brésil. A cet ensemble relativement homogène, on ajoutera les ouvrages qui donnent la parole aux Indiens d'Amérique, de Soleil hopi à De mémoire indienne, car ils complètent la vision personnelle de Jean Malaurie sur les peuples indiens, proches d'une certaine façon des Inuit auquel il a consacré également plusieurs volumes. Il convient de mentionner, pour être complets, les livres sur le shtetl d'Europe centrale, les camps de concentration - Sachso - et la Shoah - Du fond de l'abîme de Hillel Seidmann parce qu'ils nous semblent également révélateurs d'une authentique politique éditoriale élaborée au fil des années par un scientifique parcourant le monde 
et dirigeant un grand laboratoire de recherche où il recrute une partie de ses auteurs tout en étant à l'écoute de l'univers dans lequel il vit afin de ne pas passer à côté de certaines évolutions.

Le cheval d'orgueil avait fait de son auteur, mais aussi par ricochet, de son editor des personnages médiatiques, et le relais de la télévision grand public avait donné à la collection Terre Humaine une audience qu'elle n'avait jamais possédée antérieurement. Trois ans plus tard, Jean Malaurie devait décider d'imiter les Editions du Seuil et publier un Bulletin Terre Humaine destiné à faire connaître les nouveaux titres de la série et à fidéliser une clientèle de plus en plus volatile. Toutefois, c'est la proposition de Bernard de Fallois, PDG du groupe Plon-Perrin depuis 1975 et artisan du battage médiatique qui fit grimper les ventes du Cheval d'orgueil à un million d'exemplaires, qui convainquit Jean Malaurie d'oublier ses préventions contre le Poche et de se lancer dans une nouvelle aventure. Ayant obtenu d'être le véritable patron de la nouvelle collection Terre Humaine Poche, donc de conserver le pouvoir de choisir ses titres, il attaquait, à partir de 1982, le public des étudiants, de plus en plus attaché à ces seules séries bon marché de volumes et davantage préoccupé de lectures en rapport avec son univers professionnel que ne l'était la génération de ceux qui avaient 'fait' 1968. Avec ce deuxième support, la collection connut une nouvelle jeunesse, rééditant des titres qui avaient particulièrement bien marché, Tristes Tropiques, Les Derniers Rois de Thulé, Le cheval d'orgueil et L'été grec, mais y ajoutant tous les nouveaux volumes de la série. Ainsi, au lieu d'être des tentatives isolées comme l'avaient été Tristes Tropiques, L'Afrique ambiguë, Les Derniers Rois de Thulé, Les Immémoriaux et La Mort sara, les seuls volumes que Jean Malaurie avait consenti, sans pouvoir faire autrement, à faire entrer dans la collection "10-18 » entre 1962 et 1971, le passage systématique de la collection mère dans la série de sa fille va consolider la position de l'éditeur ${ }^{29}$ et lui gagner la confiance des nouveaux patrons d'un groupe d'édition qui, de 1975 à 2005, connaîtra de multiples propriétaires ${ }^{30}$.

En plus de la collection Terre Humaine Poche et des neuf Bulletins Terre Humaine qui s'échelonnent de 1978 à 1985, deux albums se sont ajoutés aux volumes - Visions d'un nomade de Wilfred Thesiger et Ultima Thulé de Jean Malaurie - ainsi que cinq ouvrages d'une série intitulée Courants de pensée - Henri Mitterand, Jacques Lacarrière, René Dumont, Jean Duvignaud et Michel Ragon - ce qui, là encore, contribue à solidifier l'assise éditoriale 
et médiatique d'une collection qui se flattera, en 2000, après quarante-cinq années d'existence, d'avoir vendu dix millions d'exemplaires ${ }^{31}$, soit, en moyenne, 222.000 volumes, chaque année, ce qui laisse évidemment rêveur et renforce le caractère mythique, ou, en tout cas, atypique, d'une série que l'on peut certes classer au rayon des sciences humaines puisque l'homme est au centre des préoccupations de tous les auteurs qui y figurent, mais qui, par ses qualités stylistiques et le ton imposé aux récits, la situe au cœur des stratégies d'écriture propres aux grandes maisons d'édition littéraire. Cette impression est renforcée par les confidences de certains auteurs qui avouent volontiers que leur directeur les a obligés à réécrire cinq ou six fois leur manuscrit, à reprendre leur travail, comme s'il s'était agi du texte d'un roman. En agissant comme n'importe quel grand editor dirigeant une maison d'édition visant la consécration littéraire, l'attribution de prix et de récompenses, des articles dans les magazines, des interviews à la radio et à la télévision, Jean Malaurie est parvenu à son but : donner à lire des hommes et des femmes, mais plutôt les premiers que les secondes, dont la vie est une leçon ou un témoignage sur un monde en train de disparaître. C'est sans doute sa grande force puisqu'il fêtera fin 2014 le soixantième anniversaire du contrat qui le lie aux éditions Plon - évidemment un record absolu dans cet univers - mais aussi ce qui achève de faire de lui un être totalement à part dans l'édition de sciences humaines.

Pour conclure ce survol consacré à l'existence étonnante d'une 'bibliothèque' scientifique, on insistera sur son ancrage incontestable dans le terreau des habitudes et des habitus propres au champ culturel français. On ne peut imaginer d'équivalent anglais ou américain à ce type de collection parce que l'organisation du champ intellectuel est différent dans ces deux pays où les presses des grandes universités sont le lieu privilégié d'accueil des travaux que Jean Malaurie a réunis dans sa collection. Bien entendu, la personnalité de 'l'editor' a joué un rôle certain dans sa réussite, sa capacité à se créer des relations dans le monde politique, médiatique ou chez les leaders d'opinion en général étant exceptionnelle. L’ancien président de la République, Jacques Chirac, était fasciné par le personnage et l'invitait volontiers à l'Elysée. L'URSS puis la Russie ont honoré l'explorateur et le défenseur inlassable de la cause des peuples du grand Nord. Les Etats-Unis, nombre d'autres pays ont également contribué à son rayonnement. Tout ceci a aidé en retour 
l'éditeur dans sa tâche, mais, au-delà de l'homme et de son immense talent, il faut admettre qu'une telle collection, Terre Humaine, sans doute la plus belle vitrine du Brésil dans le monde académique européen, ne pouvait naitre que dans un pays où le champ culturel demeure le lieu d'affrontements entre des forces qui le débordent considérablement et lui interdisent cette autonomie qui, ailleurs, le rend certes plus indépendant mais le prive de ces possibilités d'intervention dans la sphère publique qui est - ou était ? - la grande caractéristique des intellectuels français au XXe siècle. 


\section{Notas e Referências}

1 Voir Pierre AUREGAN. Des récits et des hommes. Terre Humaine : un autre regard sur les sciences de l'homme, Paris, Nathan/Plon, 2001, pour une analyse systématique de cette collection et Terre Humaine. Cinquante ans d'une collection, dir. Mauricette BERNE et Jean-Marc TERRASSE. Paris : BnF, 2005, pour une étude à multiples entrées.

2 Roger CHARTIER. L'ordre des livres. Lecteurs, auteurs, bibliothèques en Europe entre XVIe et XVIIIe siècle. Aix-en-Provence : Alinéa, 1992, p. 74.

3 Isabelle OLIVERO. L'invention de la collection : de la diffusion de la littérature et des savoirs à la formation du citoyen au XIXe siècle. Paris : IMEC Ed., 1999.

$4 \mathrm{Du}$ "poche " aux collections de poche. Histoire et mutations d'un genre, dir. JeanYves MOLLIER et Lucile TRUNEL, Liège, Céfal, Les Cahiers des paralittératures $\mathrm{n}^{\circ}$ 10, 2009.

5 Isabelle OLIVERO, op.cit.

6 Jean-Yves MOLLIER. Louis Hachette (1800-1864). Le fondateur d'un empire. Paris : Fayard, 1999.

7 Marie-Laure AURENCHE. Edouard Charton et l'invention du «Magasin pittoresque » (1833-1870). Paris : Honoré Champion, 2002.

8 Jean-Yves MOLLIER. "Un éditeur dreyfusard : Pierre-Victor Stock ». in : L'affaire Dreyfus. dir. Michel WINOCK. Paris : Seuil, 1998, p. 195-201.

9 Valérie TESNIERE. Le Quadrige. Un siècle d'édition universitaire. 1860-1968. Paris : PUF, 2001, p. 331-339.

10 Dictionnaire des intellectuels français, dir. Jacques JULLIARD et Michel WINOCK. Paris : Seuil, 2002.

11 Voir sur cette genèse les travaux de François DOSSE, notamment son Histoire du structuralisme, Paris, La Découverte, 1992, 2 vol., et La marche des idées. Histoire des intellectuels, histoire intellectuelle, Paris, La Découverte, 2003, ainsi que L'histoire des intellectuels aujourd'hui, dir. Michel LEYMARIE et JeanFrançois SIRINELLI, Paris, PUF, 2003.

12 Jean-Yves MOLLIER. "Une aventure éditoriale hors des sentiers battus ", in Terre Humaine. Cinquante ans d'une collection, op.cit., p. 129-140.

13 Jean-Yves MOLLIER. Edition, presse et pouvoir en France au XXe siècle. Paris : Fayard, 2008.

14 Ibidem. 
15 Matthieu LETOURNEUX et Jean-Yves MOLLIER. La Librairie Tallandier. Histoire d'une grande maison d'édition populaire (1870-2000). Paris : Nouveau Monde éditions, 2011, et Sylvain VENAYRE. La gloire de l'aventure. Paris : Aubier, 2002.

16 Pierre AUREGAN. op. cit.

17 Benoît MARPEAU. Gustave Le Bon. Parcours d'un intellectuel, 1841-1931. Paris : CNRS Editions, 2000.

18 Jean MALAURIE. 'Entretien avec Hélène Willemart'. Pour Jean Malaurie, Paris, Plon, 1990, p. 773 et sqs.

19 Philippe OlIVERA. 'Edition d'histoire', Historiographies. Concepts et débats. dir. Christian DELACROIX, François DOSSE, Patrick GARCIA et Nicolas OFFENSTADT. Paris, Gallimard, coll. Folio Histoire . 2010, 2 vol., T. II, p. 112-123.

20 Jean-Yves MOLLIER. Edition, presse et pouvoir en France au XXe siècle, op. cit.

21 Edith MORA. "Editeurs, qui êtes-vous ? A la Librairie Plon". In : Les Nouvelles littéraires. 27 novembre 1952, et Jean-Yves MOLLIER. op. cit., pour une étude de cette série de reportages sur l'édition française.

22 Bernard DROZ. "Soustelle, Jacques". In : Dictionnaire des intellectuels. op.cit., p. 1306-1307.

23 Jean-Yves MOLLIER. Edition, presse et pouvoir en France au XXe siècle. op. cit.

24 Ibid.

25 Ibid.

26 Jean-Claude DUBOST et François LAURENT. 'De Terre Humaine à Terre Humaine Poche'». In: Terre Humaine. Cinquante ans d'une collection, op. cit., p. 144-148.

27 Pierre Aurégan. op. cit.

28 Ibid.

29 Ibid.

30 Jean-Yves MOLLIER. op.cit.

31 Pierre AUREGAN. op. cit. 